\title{
Tumor suppressive microRNA-429 regulates cellular function by targeting VEGF in clear cell renal cell carcinoma
}

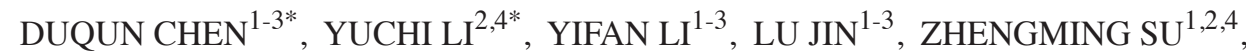 \\ ZUHU YU ${ }^{1-3}$, SHANGQI YANG ${ }^{1,2}$, XIANGMING MAO ${ }^{1,2}$ and YONGQING LAI ${ }^{1,2}$ \\ ${ }^{1}$ Department of Urology, Peking University Shenzhen Hospital; \\ ${ }^{2}$ The Guangdong and Shenzhen Key Laboratory of Male Reproductive Medicine and Genetics, \\ Peking University Shenzhen Hospital, Institute of Urology of Shenzhen PKU-HKUST Medical Center, \\ Shenzhen, Guangdong 518036; ${ }^{3}$ Department of Urology, Anhui Medical University, Hefei, Anhui 230032; \\ ${ }^{4}$ Department of Urology, Shantou University Medical College, Shantou, Guangdong 515041, P.R. China
}

Received December 7, 2014; Accepted October 14, 2015

DOI: $10.3892 / \mathrm{mmr} .2015 .4653$

\begin{abstract}
Clear cell renal cell carcinoma (ccRCC) is the predominant and most aggressive type of kidney malignancy, however, the mechanism underlying its carcinogenesis remains to be elucidated. The present study aimed to determine the expression and function of microRNA (miR)-429 in ccRCC carcinogenesis. Reverse transcription-quantitative polymerase chain reaction (RT-qPCR) was used to detect the expression of miR-429 in ccRCC specimens. Following transfection of miR-429 synthetic mimics, the expression of miR-429 was examined and cell proliferation, cell migration, apoptosis and luciferase assays were conducted in ccRCC cell lines. The results demonstrated that expression of miR-429 was decreased in ccRCC cells. In addition, upregulation of miR-429 by transfection of mimics reduced cellular proliferation and migration, and induced apoptosis in ACHN and 786-0 cell lines. Furthermore, miR-429 decreased the 3'UTR luciferase activity of vascular endothelial growth factor (VEGF) and $\mathrm{c}-\mathrm{MYC}$, and RT-qPCR analysis demonstrated that the cancer cells transfected with miR-429 mimics exhibited decreased expression of VEGF, but not c-MYC. To the best of our knowledge, the present study was the first to reveal that downregulated miR-429 functioned as a tumor suppressor by restraining cellular proliferation and migration, and inducing apoptosis, as well as targeting VEGF in ccRCC cells.
\end{abstract}

Correspondence to: Professor Yongqing Lai, Department of Urology, Peking University Shenzhen Hospital, Institute of Urology of Shenzhen PKU-HKUST Medical Center, 1120 Lianhua Road, Shenzhen, Guangdong 518036, P.R. China

E-mail: yqlord@163.com

*Contributed equally

Key words: microRNA-429, vascular endothelial growth factor, clear cell renal cell carcinoma

\section{Introduction}

Kidney cancer accounts for $\sim 3 \%$ of all novel cancer diagnoses worldwide. It is the urologic malignancy with the lowest rate of survival and has an estimated 5-year survival rate of $50-60 \%$ (1). Nearly $30 \%$ of patients with renal cell carcinoma (RCC) develop distant metastasis at initial presentation and up to $30 \%$ of patients with RCC who received traditional surgery experienced recurrence during subsequent follow-up (2). Clear cell RCC (ccRCC) is the most common (80-90\%) type of kidney cancer (3). However, the numerous tumor suppressor genes and oncogenes that are mutated and result in ccRCC remain to be elucidated (4). Global studies of copy number, gene sequencing, gene expression and miRNA expression in primary RCC may aid in identifying these genes (5).

MicroRNAs (miRNAs) are a class of noncoding RNAs, typically 20-23 nucleotides in length. They have been confirmed to be one of the most abundant groups of regulatory genes in multicellular organisms, and are important in a number of fundamental cellular processes (6). Numerous studies have used high-throughput microarrays to identify cancer-specific miRNA fingerprints in certain types of cancer (7-9), including ccRCC (10). These studies indicate that alterations in miRNAs are critical in the carcinogenesis of numerous, and perhaps all types of human cancer (11). Certain miRNAs may be directly involved in cancer development by controlling cell differentiation and apoptosis, while others may be involved by targeting cancer oncogenes and/or tumor suppressor genes (12). Understanding of the function of miRNAs is providing novel insights into the molecular basis of cancer, and biomarkers for cancer diagnoses and therapy (13).

As a member of the miR-200 family, miR-429 has been confirmed to be dysregulated in various types of human cancer, including colorectal cancer (14), gastric cancer (15) and esophageal carcinoma (16). In addition, aberrant expression of miR-429 can serve as a biomarker for the early detection and prognosis of colorectal cancer (14), non-small cell lung cancer (17) and serous ovarian carcinoma (18). However the role of miR-429 in ccRCC remains to be identified. Thus, the 
present study aimed to determine the function and possible molecular mechanisms of miR-429 in ccRCC.

\section{Materials and methods}

ccRCC clinical specimens and cancer cell lines. A total of 40 paired ccRCC specimens and adjacent normal tissues were obtained from Peking University Shenzhen Hospital (Shenzen, China) and Anhui Medical University First Affiliated Hospital (Hebei, China). The samples were collected during nephrectomy between August 2011 and July 2013, and written informed consent was obtained from the patients. Collection was in accordance with the IRB-approved protocol for human specimen collection, and for the use of these materials and associated clinical information for research purposes (19). All samples were processed and stored at $-80^{\circ} \mathrm{C}$ in RNAlater (Qiagen, Valencia, CA, USA) until RNA isolation. The clinical and pathological information of all the patients is summarized in Table I. The present study was reviewed and approved by the Ethics Committee of Peking University Shenzhen Hospital.

ACHN and 786-O human RCC cell lines, and the HeLa cervical cancer cell line were used in the present study. HeLa cells were used in the present study, as they are easily transfected and often used for luciferase reporter assay The cells were cultured in Dulbecco's modified Eagle's medium (DMEM; Sigma-Aldrich, St. Louis, MO, USA) supplemented with $10 \%$ fetal bovine serum and incubated at $37^{\circ} \mathrm{C}$ in $5 \%$ carbon dioxide.

Total RNA isolation and reverse transcription-quantitative polymerase chain reaction (RT-qPCR). Total RNA of human specimens and cells, including miRNA was extracted by TRIzol reagent (Invitrogen; Thermo Fisher Scientific, Inc., Waltham, MA, USA) according to the manufacturer's instructions. To quantify the expression of miR-429, cDNA templates obtained by miScript Reverse Transcription (Qiagen, Hilden, Germany) was used for RT-qPCR with U6 serving as an internal control. While gene expression was quantified using cDNA templates obtained by the Revert Aid First Strand cDNA Synthesis kit (MBI Fermentas, Burlington, ON, Canada). SYBR Premix Ex Taq II (Tli RNaseH Plus; Takara Biotechnology, Inc., Otsu, Japan) was used with human GAPDH serving as an endogenous control. All primers used in the present study are presented in Table II and qPCR was performed in the LightCycler 480 Real-Time PCR system (Roche, Basel, Switzerland), according to the manufacturer's instructions. PCR amplification was performed using $1 \mu \mathrm{l}$ cDNA in a $20 \mu \mathrm{l}$ reaction system, containing $10 \mu \mathrm{l}$ QuantiTect SYBR Green PCR Master mix (Qiagen, Valencia, CA, USA), $2 \mu \mathrm{l}$ miScript Universal Primer (Qiagen), $0.5 \mu \mathrm{l}$ specific microRNA primer (Invitrogen; Thermo Fisher Scientific, Inc.) and $6.5 \mu \mathrm{l}$ RNase-free water. PCR amplification conditions were set as: $95^{\circ} \mathrm{C}$ for $2 \mathrm{~min}, 40$ cycles of $95^{\circ} \mathrm{C}$ for $15 \mathrm{sec}, 58^{\circ} \mathrm{C}$ for $30 \mathrm{sec}$ and $72^{\circ} \mathrm{C}$ for $30 \mathrm{sec}$.

Cell transfection. For upregulation of miR-429, the cancer cell lines were transfected with Lipofectamine RNAiMAX transfection reagent (Invitrogen; Thermo Fisher Scientific, Inc.) and Opti-MEM (Invitrogen; Thermo Fisher Scientific, Inc.) with synthetic miR-429 mimics (Shanghai GenePharma Co.,
Table I. Clinical and pathological features of 40 patients.

\begin{tabular}{lc}
\hline Variable & Number of cases \\
\hline Total & 40 \\
Age (years) & \\
$\geq 52$ & 23 \\
$<52$ & 17 \\
Gender & \\
Male & 25 \\
Female & 15 \\
PT-stage & \\
T1 & 21 \\
T2 & 17 \\
T3 and T4 & 2 \\
AJCC clinical stage & \\
I & 21 \\
II & 16 \\
III+IV & 3 \\
\hline
\end{tabular}

PT, primary tumor; AJCC, American Joint Committee on Cancer.

Ltd., Shanghai, China). Cells were seeded in 6-well plates for apoptosis assays and RNA isolation $\left(30 \times 10^{4}\right.$ cells per well), in 12 -well plates for wound scratch assays $\left(25 \times 10^{4}\right.$ cells per well), in 24-well plates for luciferase reporter assays $\left(10 \times 10^{4}\right.$ cells per well), and in 96-well plates for cell proliferation assays ( $\sim 5,000$ cells per well). A normal control (NC), which simulated the structure of the miR mimics, exerted no effect on cells following transfection. The experiment was repeated a minimum of three times and the expression ratio of miR-429 transfected with mimics versus miR-429 transfected with NC was calculated.

Cell proliferation and migration assay. To determine the effect of miR-429 on cell proliferation, a 3-(4,5-dimethylthiazol-2-yl)-2,5-diphenyltetrazolium bromide (MTT) assay (Sigma-Aldrich) was used according to the manufacturer's protocols. At $0,24,48$ or $72 \mathrm{~h}$ after transfection for $6 \mathrm{~h}$, the cancer cells were incubated with $20 \mu \mathrm{l}$ MTT $(5 \mu \mathrm{g} / \mathrm{ml})$ for $4 \mathrm{~h}$, followed by the addition of $150 \mu \mathrm{l}$ dimethyl sulfoxide (Sigma-Aldrich) and agitating for $\sim 15 \mathrm{~min}$ at room temperature. The optical density (OD) was determined using a microplate reader (model 680; Bio-Rad, Hercules, CA, USA) at a dual wavelength of 490/630 nm.

Wound scratch assay. Cells were seeded in 12-well plates and a scratch was made with a P-20 micropipette tip. The initial length $(0 \mathrm{~h})$ and the residual gap length $24 \mathrm{~h}$ after the scratch were calculated from photomicrographs using MIAS-2000 software (Leica Microsystems GmbH, Wetzlar, Germany). All experiments were performed in triplicate and repeated at least three times.

Apoptosis assay. Flow cytometry (Beckman Coulter, Miami, FL, USA) was performed to evaluate the apoptosis rate of 
Table II. Primers for reverse transcription-quantitative polymerase chain reaction.

\begin{tabular}{lll}
\hline Name & \multicolumn{1}{c}{ Forward primer (5'-3') } & \multicolumn{1}{c}{ Reverse primer $\left(5^{\prime}-3^{\prime}\right)$} \\
\hline miR-429 & TAATACTGTCTGGTAAAACCGT & Provided by the miScript SYBR Green PCR kit \\
U6 & CTCGCTTCGGCAGCACA & ACGCTTCACGAATTTGCGT \\
VEGF & CACCATTGAAACCACTAGTT & GAGAGATTTAGTATGTAGAATTCTC \\
c-MYC & GGAAAAGTAAGGAAAACGATTC & AAGATTTGGCTCAATGATATATTTG \\
GAPDH & AGAAGGCTGGGGCTCATTTG & AGGGGCCATCCACAGTCTTC
\end{tabular}

miR, microRNA; VEGF, vascular endothelial growth factor.
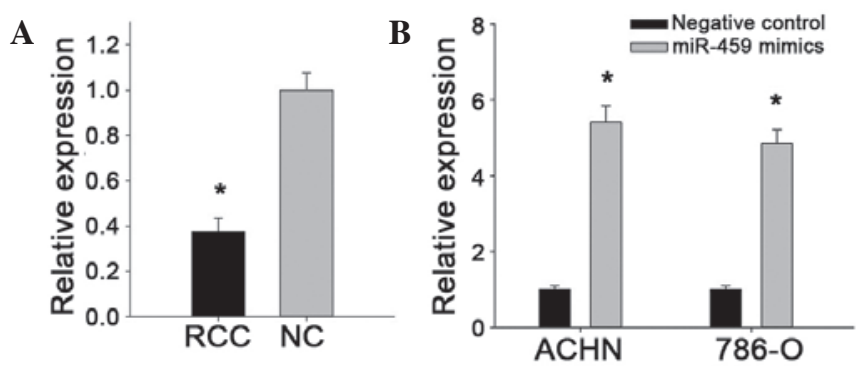

Figure 1. Reverse transcription-quantitative polymerase chain reaction to identify miR-429 expression in RCC. (A) Relative expression of miR-429 in normal tissues and RCC tissues. (B) miR-429 expression in ACHN and 786-O cells $24 \mathrm{~h}$ following transfection with miR-429 mimics. Error bars demonstrate the differences between a minimum of three experiments. ${ }^{*} \mathrm{P}<0.05$. NC, normal control; RCC, renal cell cancer.

cancer cells following transfection. Cancer cells including floating cells were harvested $48 \mathrm{~h}$ after transfection, washed twice with cold phosphate-buffered saline (PBS) and resuspended in $100 \mu 1 \mathrm{X}$ binding buffer (Invitrogen; Thermo Fisher Scientific, Inc.), followed by the addition of $5 \mu 1$ of Annexin V-fluorescein isothiocyanate (Invitrogen; Thermo Fisher Scientific, Inc.) and $5 \mu \mathrm{l}$ propidium iodide (Invitrogen; Thermo Fisher Scientific, Inc.). The fluorescence of stained cells was then analyzed by flow cytometry (Beckman Coulter, Brea, CA, USA) using $488 \mathrm{~nm}$ excitation within $30 \mathrm{~min}$ after staining, according to the manufacturer's instructions.

Bioinformatics. The potential targets of miR-429 were predicted by combining results from TargetScan (http://www.targetscan. org/), PicTar (http://pictar.mdc-berlin.de/), miRanda (http://www.targetscan.org/) and miRWalk (http://www. umm.uni-heidelberg.de/apps/zmf/mirwalk/). Putative genes predicted by all the four algorithms were accepted and candidates were selected based on the gene function.

Plasmid construction and luciferase reporter assay. The miRNA target sequences were inserted between the XhoI-NotI restriction sites in the 3'-untranslated region (UTR) of the target gene in the psiCHECK-2 vector (Promega Corporation, Madison, WI, USA), generating the wide type psiCHECK2-3'UTR (Wt). The mutant type (Mt) was generated by changing the putative binding site to 5'-AATACTG-3' in the complementary site for the seed region of miR-429. All constructed plasmids were sequence-verified by DNA sequencing analysis.

HeLa cells were transfected with $200 \mathrm{ng}$ vector, 40 pmol miRNA and $2.5 \mu 1$ Lipofectamine (Invitrogen; Thermo Fisher
Scientific, Inc.) in $100 \mu \mathrm{l}$ opti-MEM (Invitrogen; Thermo Fisher Scientific, Inc.) in 24-well plates. Luciferase assays were performed using a luciferase assay kit (Promega Corporation) according to the manufacturer's protocol. The activities of firefly and Renilla luciferase in the cell lysates were determined with a dual-luciferase assay system.

Statistical analysis. All statistical analysis was conducted with SPSS 17.0 statistical software package (SPSS Inc., Chicago, IL, USA). The difference in expression of miR-429 in ccRCC and paired normal samples was analyzed by a paired t-test. $\mathrm{P}<0.05$ was considered to indicate a statistically significant difference.

\section{Results}

Downregulation of miR-429 in ccRCC quantified by RT-qPCR. miR-429 has been confirmed to be dysregulated in numerous types of cancer (14-16); however, its expression in RCC remains unclear. In the present study, RT-qPCR was used to quantify the expression of miR-429 in 40 paired ccRCC specimens and normal specimens. The results showed that miR-429 was downregulated in 33/40 ccRCC specimens, with an average of 0.3737-fold reduction in expression (Fig. 1A). The decreased expression of miR-429 was concordant with the results of a recent miRNA expression profile study of ccRCC (10).

To analyze the effect of miR-429 on renal cancer cells, synthetic miR-429 mimics were transfected into ACHN and 786-O cancer cell lines for the gain-of-function experiments. The fold change of miR-429 expression in ACHN and 786-O cells after transfection were 5.1627 and 4.8768 quantified by RT-qPCR, respectively, as presented in Fig. 1B 

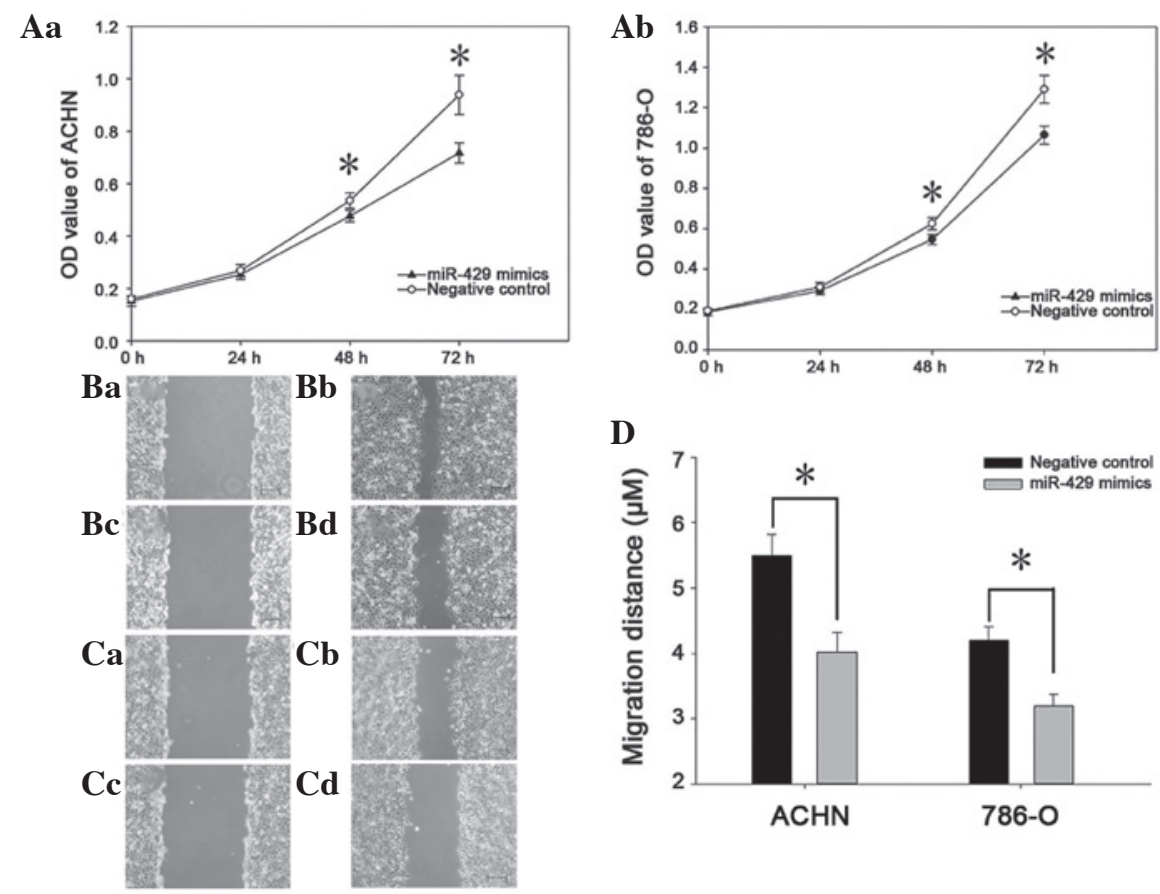

Figure 2.3-(4,5-dimethylthiazol-2-yl)-2,5-diphenyltetrazolium bromide assay for cell proliferation of ACHN and 786-O cells transfected with miR-429 mimics or negative control. Cell proliferation of (Aa) ACHN and (Ab) 786-O cells. (B and C) Wound scratch assay for ACHN and 786-O cells $24 \mathrm{~h}$ after transfection. Images of the ACHN cells transfected with miR-429 mimics at $(\mathrm{Ba}) 0$ and $(\mathrm{Bb}) 24 \mathrm{~h}$ after the wounds were made at the same point. Images of the ACHN cells transfected with negative control $(\mathrm{Bc}) 0$ and $(\mathrm{Bd}) 24 \mathrm{~h}$ after the wounds were made. Images of the 786-O cells transfected with miR-429 mimics $(\mathrm{Ca}) 0$ and (Cb) $24 \mathrm{~h}$ after the wounds were made at the same point. Images of the 786-O cells transfected with negative control $(\mathrm{Cc}) 0$ and $(\mathrm{Cd}) 24 \mathrm{~h}$ after the wounds were made. (D) Comparison of migration distance $(\mu \mathrm{m})$ in ACHN and 786-O cells using a standard caliper. "P<0.05. miR, microRNA; OD, optical density.

Aa

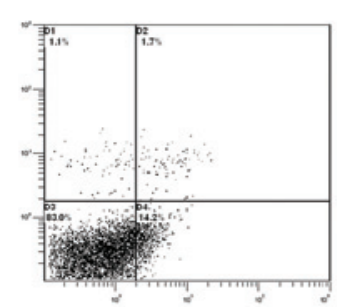

Ba

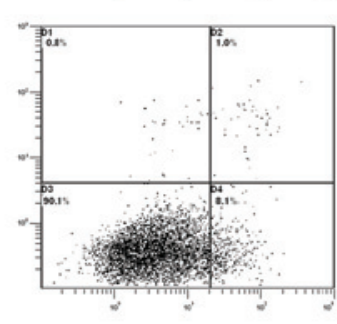

Ab

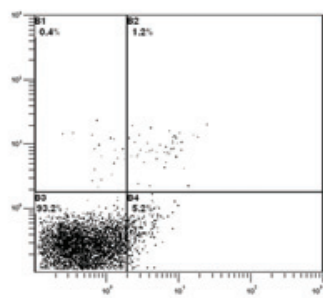

Bb

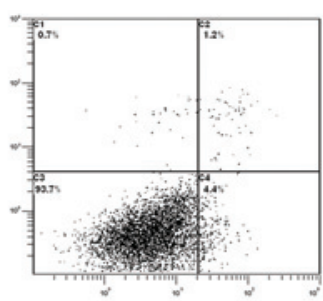

C

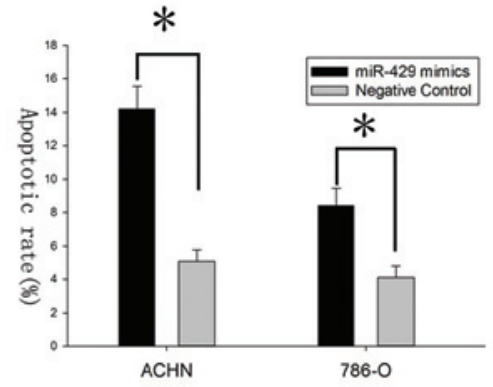

Figure 3. Flow cytometry for cell apoptosis of ACHN and 786-O cells after transfection with miR-429 mimics or negative control. ACHN cells transfected with (Aa) miR-429 mimics or (Ab) negative control. 786-O cells transfected with ( $\mathrm{Ba})$ miR-429 mimics or $(\mathrm{Bb})$ negative control. (C) Comparison of cell apoptosis rates transfected with miR-429 mimics and negative control. ${ }^{*} \mathrm{P}<0.05$. miR, microRNA.
Restoration of miR-429 inhibits cell proliferation and migration. The impact of miR-429 on cell proliferation was determined by an MTT assay, the OD value of the two groups (miR-429 mimics and negative control) was measured $0,24,48$ and $72 \mathrm{~h}$ after transfection. The results showed that the proliferation of ACHN cells decreased by 5.76, 10.48 and $20.86 \%(\mathrm{P}<0.05)$, while the proliferation of $786-\mathrm{O}$ cells decreased by $6.02,11.65$ and $26.12 \%(\mathrm{P}<0.05$; Fig. 2A). Wound scratch assays were used to evaluate the migration ability of cancer cells. As presented in Fig. 2, the wound width in the group transfected with miR-429 mimics was greater than that of the negative control group $(\mathrm{P}<0.05)$. These results indicate that miR- 429 can restrain the proliferation and migration of renal cancer cells.

miR-429 mimics induce cell apoptosis. To demonstrate the effect of miR-429 on cell apoptosis, flow cytometry was performed to detect the apoptosis rates of ACHN and 786-O cells after transfection. The results revealed that apoptosis rates of ACHN cells transfected with miR-429 mimics and those in the negative control group were 14.2 vs. $5.2 \%$ while the apoptosis rates of $786-\mathrm{O}$ cells were 8.1 vs. $4.4 \%\left({ }^{*} \mathrm{P}<0.05\right)$, suggesting that miR-429 could induce the apoptosis of renal cancer cells (Fig. 3).

miR-429 targets vascular endothelial growth factor (VEGF) in $c c R C C$. To investigate the potential target genes of miR-429, TargetScan, PicTar, miRanda and miRWalk were combined to predict the putative downstream genes. VEGF and c-MYC were two of the target genes predicted by all four algorithms. The 3'UTR of the two genes contained a complementary site for the seed sequences of miR-429 (Fig. 4A). 

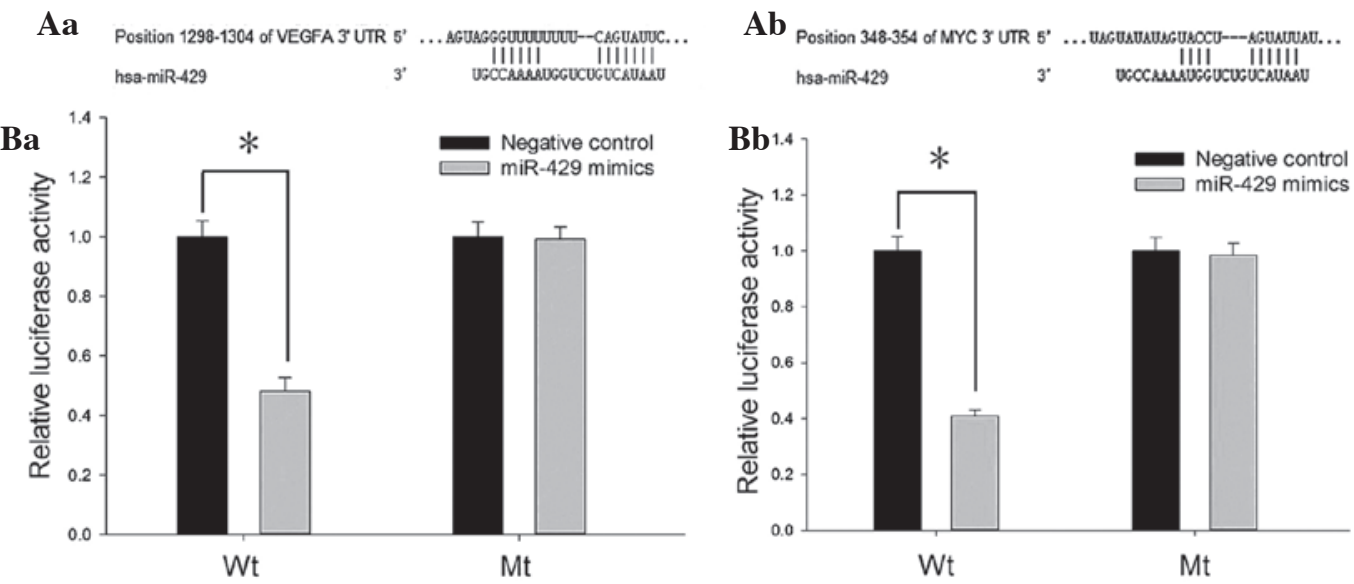

Figure 4. (Aa) Complementary sites for miR-429 and VEGF and (Ab) miR-429 and c-MYC as predicted by TargetScan. Luciferase reporter assay for HeLa cells following co-transfection with the constructed plasmids containing (Ba) VEGF and (Bb) c-MYC, and miR-429 mimics or negative control. All luciferase assays were performed in triplicate and measurements were obtained $24 \mathrm{~h}$ after transfection. miR, microRNA; VEGF, vascular endothelial growth factor; UTR, untranslated region; Wt, wild type; Mt, mutant type. ${ }^{*} \mathrm{P}<0.05$.

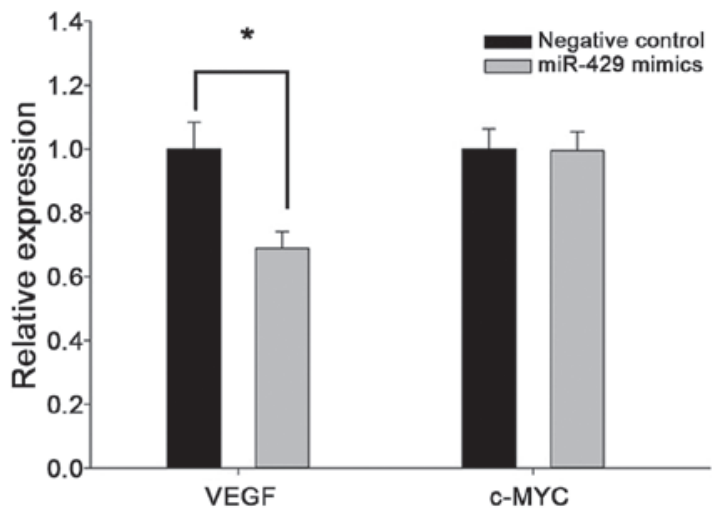

Figure 5. Relative expression of VEGF and c-MYC following transfection with miR-429 mimics or negative control. " $\mathrm{P}<0.05$. VEGF, vascular endothelia; growth factor; miR, microRNA.

To determine whether VEGF and c-MYC were directly regulated by miR-429, the 3'UTR of the two genes containing the putative binding site or mutant binding site were cloned into psiCHECK-2 to construct recombinant plasmids (Wt and $\mathrm{Mt}$ ), and the luciferase reporter assay was performed in $\mathrm{HeLa}$ cells. As presented in Fig. 4B, the relative luciferase activity of Wt recombined plasmids (containing 3'UTR of VEGF or c-MYC) was significantly decreased when transfected with miR-429 mimics $(\mathrm{P}<0.05)$, while no notable reduction was observed in the mutant groups.

In addition, RT-qPCR analysis of cancer cells transfected with miR-429 mimics showed decreased expression of VEGF (Fig. 5), but not c-MYC. These findings strongly indicate that VEGF is a direct target gene of miR-429 in renal caner, but whether c-MYC is also a target of miR-429 requires further investigation.

\section{Discussion}

MicroRNAs (miRNAs) are small endogenous non-coding RNAs that exhibit important regulatory roles via the RNA-interference pathway by targeting mRNAs for cleavage or translational repression (20); hence, decreasing the expression of the resulting protein (21). A number of experiments have shown that miRNAs could function as important regulators in the carcinogenesis of renal cancer. For example, miR-133b inhibited cell proliferation, migration and invasion by targeting matrix metallopeptidase-9 (22), and downregulating miR-501-5p induced increases in caspase-3 activity and p53 expression, as well as decreasing mTOR activation, which leads to the stimulation of the apoptotic pathway (23). In addition, miRNAs may also be promising biomarkers for RCC diagnosis. Vergho et al (24) confirmed the combination of expression levels of miR-21 and miR-126 is associated with cancer-specific survival in ccRCC, and downregulation of microRNA501-5p promotes a good prognosis (23). However, the expression and function of $\mathrm{miR}-429$ in ccRCC remains to be determined.

In the present study, RT-qPCR was used to quantify the expression of miR-429 in 48 paired ccRCC specimens and normal specimens, and the function of miR-429 on cellular proliferation, migration and apoptosis were analyzed by an MTT assay, a wound scratch assay and an apoptosis assay, respectively. The results demonstrated that miR-429 was downregulated in ccRCC. Upregulation of miR-429 by synthetic mimics restrained cellular proliferation and migration, and induced apoptosis, indicating that miR-429 may act as a tumor suppressor in ccRCC. To explore the potential target genes of miR-429, TargetScan, PicTar, miRanda and miRWalk were combined to predict the putative downstream genes, and VEGF and c-MYC were selected. Furthermore, miR-429 decreased the 3'UTR luciferase activity of VEGF and c-MYC. In addition, RT-qPCR analysis of cancer cells transfected with miR-429 mimics showed decreased expression of VEGF, but not c-MYC. All these findings strongly indicated that VEGF was a direct target gene of miR-429 in renal caner, but whether c-MYC was also the target of miR-429 remains to be explored.

ccRCC is the predominant and most aggressive subtype of kidney cancer, which is associated with a high rate of recurrence and mortality. Inactivation of the von Hippel-Lindau (VHL) gene leads to increased levels of hypoxia-inducible factor (HIF) and overexpression of HIF target genes, such as VEGF, 
CCND1, ANGPTL4 and GLUT1 (25), which exhibit an important role in the carcinogenesis of ccRCC (26). Advances in the knowledge of the role of VEGF in tumor angiogenesis, growth and progression have permitted development of approaches for the treatment of metastatic RCC (mRCC), including several agents that target VEGF and VEGF receptors (27). Currently, available oral VEGF tyrosine kinase inhibitors approved for the treatment of mRCC include sorafenib, sunitinib, pazopanib and axitinib (28). In addition, the MYC pathway was demonstrated to be activated in ccRCC and essential for the proliferation of ccRCC cells (29). Anti-VEGF therapy has been widely used in the treatment of mRCC, as VEGF can be downregulated by miR-429. Therefore, targeting miRs may present as a novel therapeutic option for mRCC treatment, via the downregulation of VEGF expression.

As described above, miRNAs regulate gene expression predominantly by translational repression, and partly, by causing target gene mRNA cleavage. As shown in the current study, decreased expression ( $\sim 30 \%$ decrease) of VEGF was observed following the transfection of miR-429 mimics, and no change in c-MYC expression was identified. This may be because miR-429 could target VEGF mRNA for cleavage and translational repression simultaneously, but could only inhibit the translation of c-MYC mRNA. Further investigation, such as gene function experiments and western blot analysis are required in order to demonstrate the association between miR-429 and VEGF and c-MYC.

In conclusion, to the best of our knowledge, the present study was the first to reveal that downregulation of miR-429 was tumor suppressive by restraining cellular proliferation and migration, inducing apoptosis, and targeting VEGF in ccRCC. The correlation between miR-429 and c-MYC, and the potential use of miR-429 in mRCC target therapy requires further investigation.

\section{Acknowledgements}

The present study was supported by the National Natural Science Foundation of China (grant no. 81101922), the Science and Technology Development Fund Project of Shenzhen (grant nos. JCYJ20130402114702124 and JCYJ20150403091443329) and the Guangdong Key Medical Subject fund.

\section{References}

1. Wulfken LM, Moritz R, Ohlmann C, Holdenrieder S, Jung V, Becker F, Herrmann E, Walgenbach-Brünagel G, von Ruecker A, Müller SC and Ellinger J: MicroRNAs in renal cell carcinoma: Diagnostic implications of serum miR-1233 levels. PLoS One 6: e25787, 2011.

2. Cho E, Adami HO and Lindblad P: Epidemiology of renal cell cancer. Hematol Oncol Clin North Am 25: 651-665, 2011.

3. Cohen HT and McGovern FJ: Renal-cell carcinoma. N Engl J Med 353: 2477-2490, 2005.

4. Keefe SM, Nathanson KL and Rathmell WK: The molecular biology of renal cell carcinoma. Semin Oncol 40: 421-428, 2013.

5. Cairns P: Renal cell carcinoma. Cancer Biomark 9: 461-473, 2010.

6. Koscianska E, Baev V, Skreka K, Oikonomaki K, Rusinov V, Tabler M and Kalantidis K: Prediction and preliminary validation of oncogene regulation by miRNAs. BMC Mol Biol 8: 79, 2007.

7. Schmidt U and Begley CG: Cancer diagnosis and microarrays Int J Biochem Cell Biol 35: 119-124, 2003.
8. Yu X, Zhang X, Bi T, Ding Y, Zhao J, Wang C, Jia T, Han D, Guo G, Wang B, et al: MiRNA expression signature for potentially predicting the prognosis of ovarian serous carcinoma. Tumour Biol 34: 3501-3508, 2013.

9. Walter BA, Valera VA, Pinto PA and Merino MJ: Comprehensive microRNA profiling of prostate cancer. J Cancer 4: 350-357, 2013.

10. Osanto S, Qin Y, Buermans HP, Berkers J, Lerut E, Goeman JJ and van Poppel H: Genome-wide microRNA expression analysis of clear cell renal cell carcinoma by next generation deep sequencing. PLoS One 7: e38298, 2012.

11. Aqeilan RI, Calin GA and Croce CM: miR-15a and miR-16-1 in cancer: Discovery, function and future perspectives. Cell Death Differ 17: 215-220, 2010.

12. Shenouda SK and Alahari SK: MicroRNA function in cancer: Oncogene or a tumor suppressor? Cancer Metastasis Rev 28: 369-378, 2009.

13. Zhang B, Pan X, Cobb GP and Anderson TA: microRNAs as oncogenes and tumor suppressors. Dev Biol 302: 1-12, 2007.

14. Sun Y, Shen S, Tang H, Xiang J, Peng Y, Tang A, Li N, Zhou W, Wang Z, Zhang D, Xiang B, et al: miR-429 identified by dynamic transcriptome analysis is a new candidate biomarker for colorectal cancer prognosis. OMICS 18: 54-64, 2014.

15. Sun T, Wang C, Xing J and Wu D: miR-429 modulates the expression of c-myc in human gastric carcinoma cells. Eur $\mathbf{J}$ Cancer 47: 2552-2559, 2011.

16. Wang Y, Li M, Zang W, Ma Y, Wang N, Li P, Wang T and Zhao G: MiR-429 up-regulation induces apoptosis and suppresses invasion by targeting Bcl-2 and SP-1 in esophageal carcinoma. Cell Oncol (Dordr) 36: 385-394, 2013.

17. Zhu W, He J, Chen D, Zhang B, Xu L, Ma H, Liu X, Zhang Y and Le H: Expression of miR-29c, miR-93, and miR-429 as potential biomarkers for detection of early stage non-small lung cancer. PLoS One 9: e87780, 2014.

18. Nam EJ, Yoon H, Kim SW, Kim H, Kim YT, Kim JH, Kim JW and Kim S: MicroRNA expression profiles in serous ovarian carcinoma. Clin Cancer Res 14: 2690-2695, 2008.

19. Levine RJ. An IRB-approved protocol on the use of human fetal tissue. IRB 11: 7-8, 1989.

20. Yoon S and De Micheli G: Prediction of regulatory modules comprising microRNAs and target genes. Bioinformatics 21 (Suppl 2): ii93-ii100, 2005.

21. Wang Y, Gu J, Roth JA, Hildebrandt MA, Lippman SM, Ye Y, Minna JD and Wu X: Pathway-based serum microRNA profiling and survival in patients with advanced stage non-small cell lung cancer. Cancer Res 73: 4801-4809, 2013.

22. Wu D, Pan H, Zhou Y, Zhou J, Fan Y and Qu P: microRNA-133b downregulation and inhibition of cell proliferation, migration and invasion by targeting matrix metallopeptidase- 9 in renal cell carcinoma. Mol Med Rep 9: 2491-2498, 2014.

23. Mangolini A, Bonon A, Volinia S, Lanza G, Gambari R, Pinton P, Russo GR, Del Senno L, Dell'Atti L and Aguiari G: Differential expression of microRNA501-5p affects the aggressiveness of clear cell renal carcinoma. FEBS Open Bio 4: 952-965, 2014.

24. Vergho D, Kneitz S, Rosenwald A, Scherer C, Spahn M, Burger M, Riedmiller $\mathrm{H}$ and Kneitz B: Combination of expression levels of miR-21 and miR-126 is associated with cancer-specific survival in clear-cell renal cell carcinoma. BMC Cancer 14: 25, 2014.

25. Zhang T, Niu X, Liao L, Cho EA and Yang H: The contributions of HIF-target genes to tumor growth in RCC. PLoS One 8: e80544, 2013.

26. Choueiri TK, Fay AP, Gagnon R, Lin Y, Bahamon B, Brown V, Rosenberg JE, Hutson TE, Baker-Neblett KL, Carpenter C, et al: The role of aberrant VHL/HIF pathway elements in predicting clinical outcome to pazopanib therapy in patients with metastatic clear-cell renal cell carcinoma. Clin Cancer Res 19: 5218-5226, 2013.

27. Conti A, Santoni M, Amantini C, Burattini L, Berardi R, Santoni G, Cascinu S and Muzzonigro G: Progress of molecular targeted therapies for advanced renal cell carcinoma. Biomed Res Int 2013: 419176, 2013.

28. Gupta S and Spiess PE: The prospects of pazopanib in advanced renal cell carcinoma. Ther Adv Urol 5: 223-232, 2013.

29. Tang SW, Chang WH, Su YC, Chen YC, Lai YH, Wu PT, Hsu CI, Lin WC, Lai MK and Lin JY: MYC pathway is activated in clear cell renal cell carcinoma and essential for proliferation of clear cell renal cell carcinoma cells. Cancer Lett 273: 35-43, 2009. 